\title{
High Performance Infrared Narrow Band Pass Filters for Infrared Sensors and Systems
}

\author{
Jing Lu \\ (jl@mirhz.com) \\ Multi IR Optoelectronics Co., Ltd . \\ Mailing Address: Building\#2, No.2,Xiyuansi Rd, Xihu T\&E Zone, Hangzhou, China, 310030.
}

\begin{abstract}
As one of the key elements, the performance of infrared narrow band pass filters affects the quality of infrared sensors and systems directly. As examples, three kinds of infrared narrow band pass filters which are used in NIR, MIR \& FIR regions respectively are introduced, including the design, manufacture process and measurement results of such high performance infrared narrow band pass filters.
\end{abstract}

Key Words: infrared sensors and systems, high performance, infrared narrow band pass filter, manufacture

Topic Number: I.4 (Infrared Optical Filters)

\section{Introduction}

Infrared band pass filters are widely used in various science and technology fields. For infrared sensor and system applications, it is often required that optical function presents reflectance and transmittance performances over separate spectral ranges from $1 \mu \mathrm{m}$ to more than $20 \mu \mathrm{m}$. Obviously, there is no material which can be used in so wide region. Usually fewer layers are needed to obtain a given contrast and the greater the width of the rejection zone. By virtue of the high refractive index in the infrared region, the use of semiconductor materials such as $\mathrm{Si}, \mathrm{Ge}$ in combinations with low index materials such as $\mathrm{ZnS}$, ZnSe, $\mathrm{SiO}, \mathrm{YbF} 3, \mathrm{YF} 3$ are of interest because it allows a reduction of the number of layers on the substrate of $\mathrm{Si}$, $\mathrm{Ge}$, sapphire,CaF2, glass and so on.

Consequently, decrease the risk of crack formation in the stacks is also of interest because there are so many thick layers in the multi-cavity infrared filter structure. Not only the filter design and employing materials, but also new evaporation process are important for filter durability. Using IAD technique, the filters can be much more robust since the films become dense and even, sometimes, can compensate the stress.

In this paper, we report on the design, preparation and characterization of three kinds of infrared narrow band pass filters which are used in NIR, MIR \& FIR regions and deposited by electron beam gun. A new design method is put forward. It can be seen that the measured optical performance of the filters has very good results in accordance with theoretical ones.

\section{Design}

The transmission curve of the basic Fabry-Perot filter is not of ideal shape. It can be proved that one half of the energy transmitted in any order lies outside the half-width. A more nearly rectangular curve would be a great improvement. If two or more of these filters are placed in series, which are called as double cavity or multiple cavity filters, a much more promising shape than the single cavity filter can be obtained. Fig.1 shows the transmittance curses of one-, two- and three-cavity filters with materials of $\mathrm{Si} / \mathrm{SiO}$ on the substrate of Glass. It can be seen that three-cavity filter has good rectangular shape. 


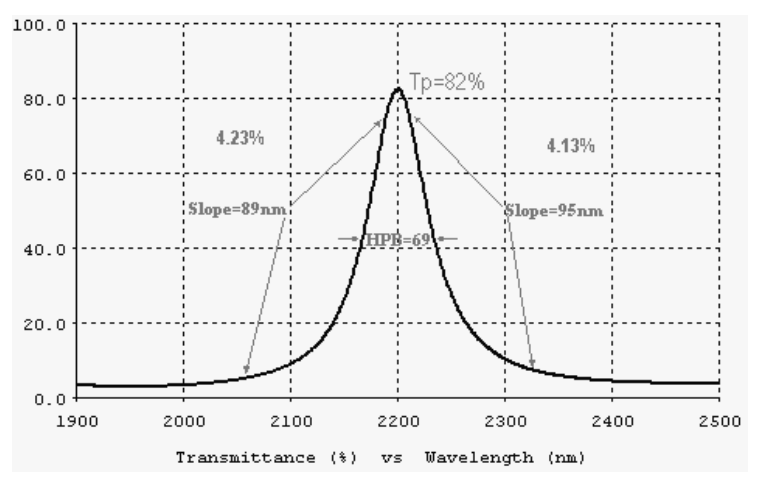

(a). One-Cavity Design ( HLH6LHLHL )

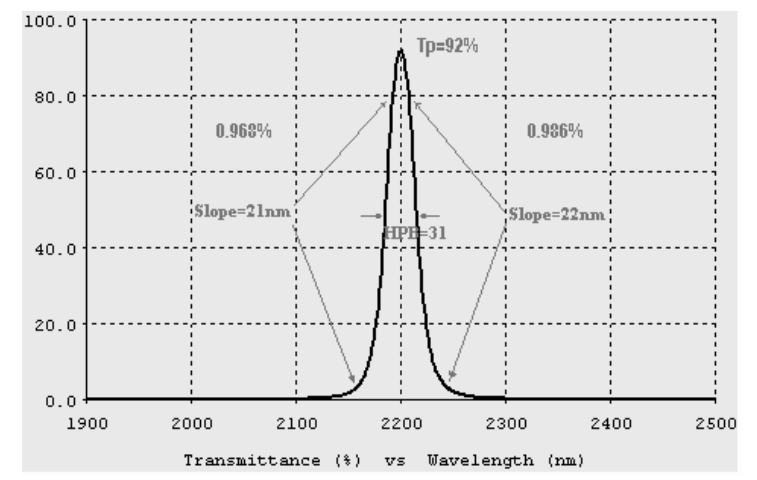

(b). Two-Cavity Design ( HLH6LHLHL HLH6LHLHL )

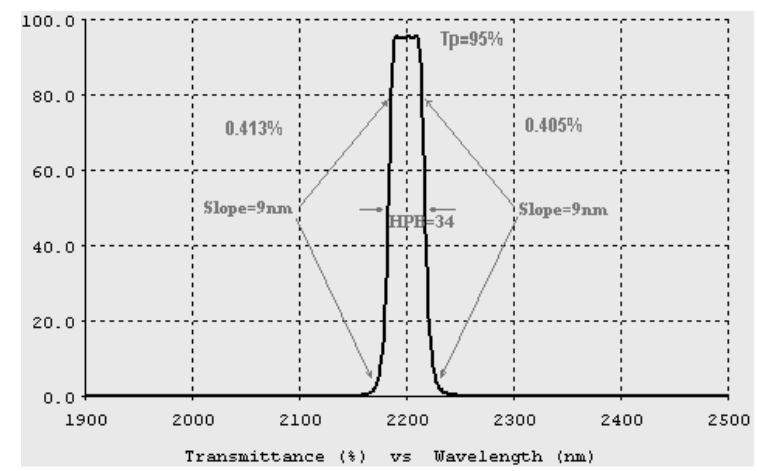

(c). Three-Cavity Design ( HLH6LHLHL HLH6LHLHL HLH6LHLHL)

Fig.1 The transmittance curses of one-, two- and three-cavity filters with central wavelength of $2200 \mathrm{~nm}$

To get more square shape of the band pass of a filter, the more cavities is needed. However, in this case, the 'rabbit's ears', or the rather prominent peaks in the band pass are inclined to occur. The reason for this problem is the loss matching of the equivalent admittance $E$ of the symmetrical period to the surrounding media. This implies that the period can, itself, be used as a simple matching assembly. So we can straightforwardly use symmetrical period method to match the admittance between symmetrical period and substrate or air. For the typical design, the filter can be written as

Sub/ $\left[(\mathrm{HL})^{\mathrm{p}}(2 \mathrm{H})^{\mathrm{q}}(\mathrm{LH})^{\mathrm{p}} \mathrm{L}\right]^{\mathrm{m}} / \mathrm{Air} \quad$ or $\mathrm{Sub} /(\mathrm{HL})^{\mathrm{p}}{ }_{0}(2 \mathrm{H})^{\mathrm{q}}{ }_{1}(\mathrm{LH})^{\mathrm{p}}{ }_{1} \mathrm{~L}(2 \mathrm{H})^{\mathrm{q}}{ }_{2}(\mathrm{LH})^{\mathrm{p}}{ }_{2} \mathrm{~L}$.../Air

Sub/ $\left[(\mathrm{HL})^{\mathrm{p}} \mathrm{H}(2 \mathrm{~L})^{\mathrm{q}} \mathrm{H}(\mathrm{LH})^{\mathrm{p}} \mathrm{L}\right]^{\mathrm{m}} /$ Air or Sub/ $(\mathrm{HL})^{\mathrm{p}}{ }_{0} \mathrm{H}(2 \mathrm{~L})^{\mathrm{q}}{ }_{1} \mathrm{H}(\mathrm{LH})^{\mathrm{p}}{ }_{1}(2 \mathrm{~L})^{\mathrm{q}}{ }_{2} \mathrm{H}(\mathrm{LH})^{\mathrm{p}}{ }_{2} \mathrm{~L}$.../Air

where $p, q$ and $m$ are periods of the stakes; $H, L$ are high and low refractive index respectively. Obviously, if the $L$ near air is cancelled, equations (1) and (2) are symmetrical periods. Therefore it is easy to calculate the equivalent admittance. As an example, supposing that $m=3, n_{H}=2.05$ and $n_{L}=1.47$, the calculated equivalent index of symmetrical periods is shown in Table 1. 
Table 1. Equivalent index of symmetrical periods

\begin{tabular}{|c|c|c|c|c|c|}
\hline $\mathrm{q}_{1}-\mathrm{q}_{2}-\mathrm{q}_{3}$ & $\begin{array}{l}\text { Equivalent } \\
\text { index }\end{array}$ & $\mathrm{q}_{1}-\mathrm{q}_{2}-\mathrm{q}_{3}$ & $\begin{array}{l}\text { Equivalent } \\
\text { index }\end{array}$ & $\mathrm{q}_{1}-\mathrm{q}_{2}-\mathrm{q}_{3}$ & $\begin{array}{l}\text { Equivalent } \\
\text { index }\end{array}$ \\
\hline $2-2-2$ & 2.07 & $4-2-4$ & 2.34 & $6-2-6$ & 2.56 \\
$2-4-2$ & 1.82 & $4-4-4$ & 2.07 & $6-4-6$ & 2.28 \\
$2-6-2$ & 1.65 & $4-6-4$ & 1.87 & $6-6-6$ & 2.07 \\
$2-8-2$ & 1.52 & $4-8-4$ & 1.72 & $6-8-6$ & 1.90 \\
\hline
\end{tabular}

It can be seen that 1). $E=2.07$, when spacer of three-cavity is in same order; 2 ). $E$ increases with second-cavity order decrease. If $\mathrm{q}_{1}-\mathrm{q}_{2}-\mathrm{q}_{3}$ is chosen as $2-8-2$, then $\mathrm{E}=1.52$, which is matched to glass substrate perfectly. Designs arrived at in this way will be satisfactory for a wide range of applications where ripple and optical loss within the band pass must be small. However, there are applications where even the performance is inadequate, in this case, the filter should also be matched to air, and so there is an extra double layer anti-reflective coating next to the incident medium.

Using similar method the filters used in wavelength of $4260 \mathrm{~nm}$ and $7300 \mathrm{~nm}$ can be constructed, as shown in Fig. 2 and 3.

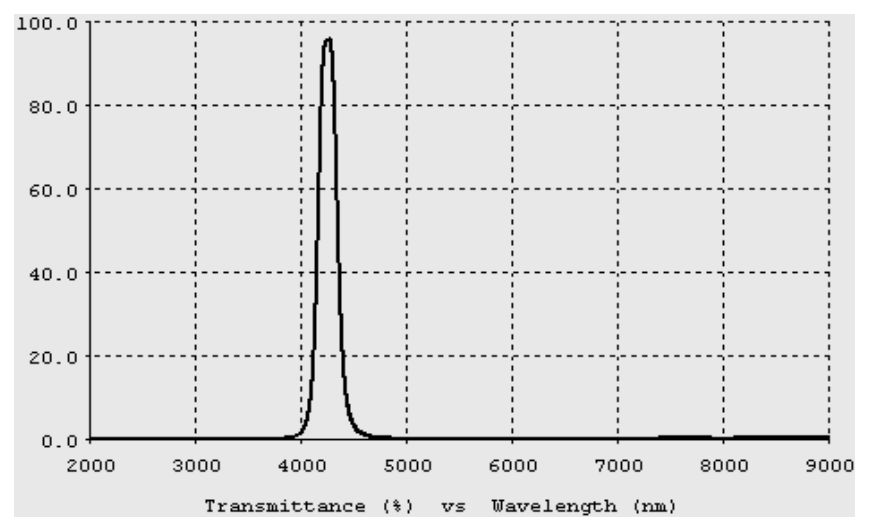

Fig.2 The transmittance curse of the filter with central wavelength of $4260 \mathrm{~nm}$

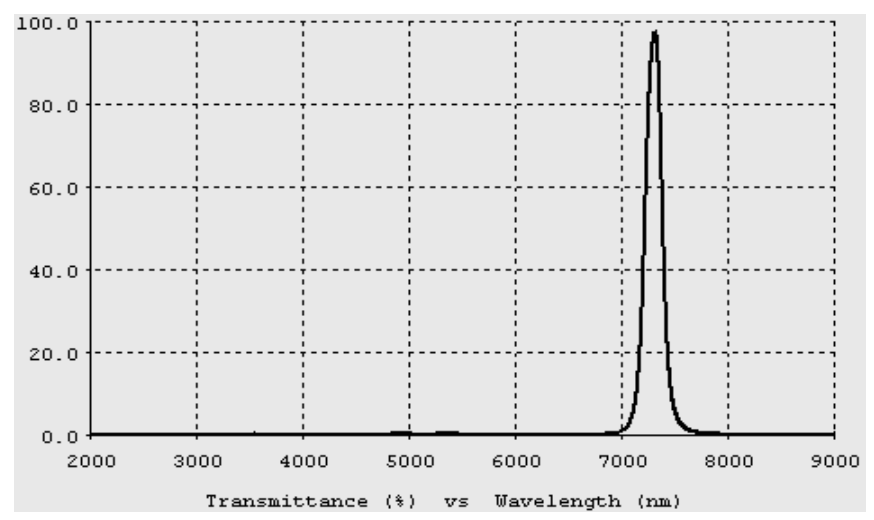

Fig.3 The transmittance curse of the filter with central wavelength of $7300 \mathrm{~nm}$

\section{Manufacture}

The filters are deposited in a box-coating system (Model: Super IR-1300, supplied by OptoRun, Modify by Multi IR). The main manufacture process is listed in Fig.4. 


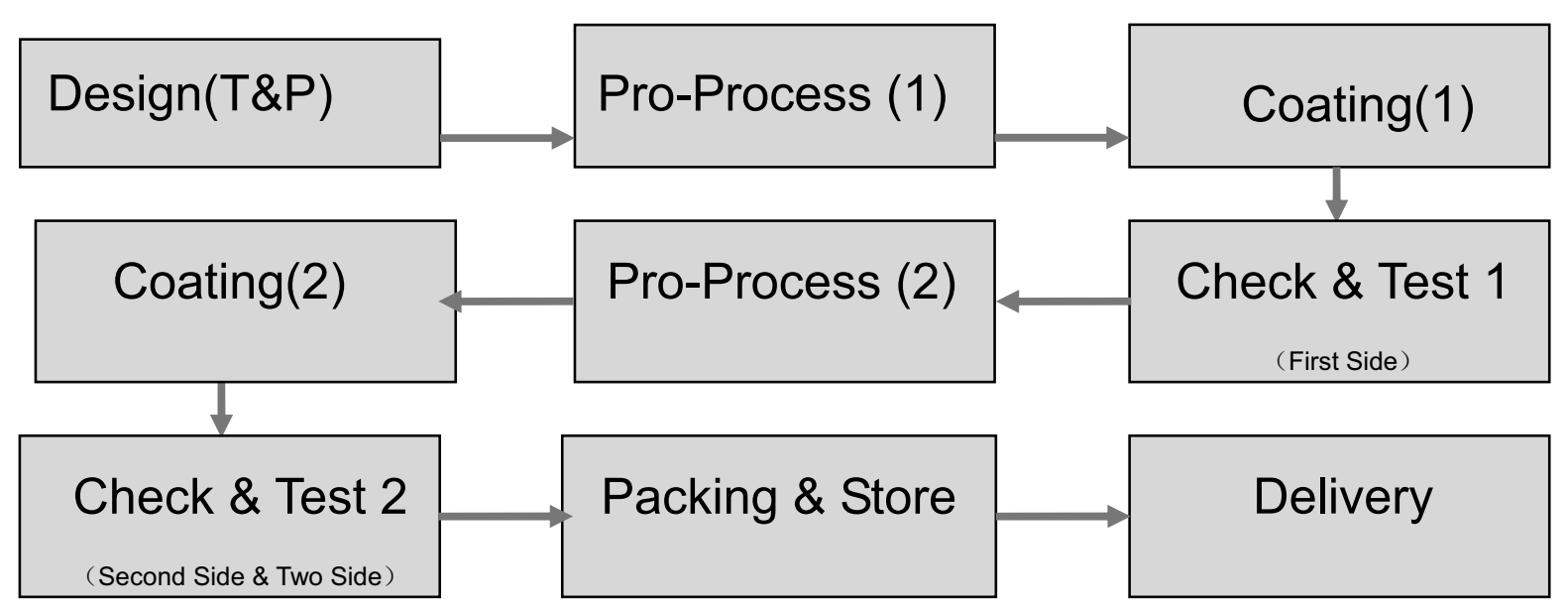

Fig.4 Main manufacture process

All the samples were deposited by the same electron beam evaporation system which was pumped down to a base pressure of less than $4 \times 10^{-3} \mathrm{~Pa}$ before evaporation. All the substrates were cleaned by alcohol and aether mixture solution. The deposition rate was controlled by a quartz monitor at $0.5-2 \mathrm{~nm} / \mathrm{s}$. The substrate temperature was $50^{\circ} \mathrm{C} \sim 300^{\circ} \mathrm{C}$ with control accuracy of $\pm 10^{\circ} \mathrm{C}$.

The thicknesses were controlled by optical monitor and quartz monitor. For quarter wave multilayer stacks, for which all optical thicknesses are equal or a multiple of $\lambda_{0} / 4$, the direct optical monitoring is known to be efficient as it allows automatic error compensation. In particular, this feature has been widely demonstrated for the production of multiple cavity filters. A monitoring apparatus dedicated for infrared range has wavelength accuracy and reproducibility of less than $\pm 2.0 \mathrm{~nm}$ and $\leq 0.2 \mathrm{~nm}$ respectively and monochromaticity of less than $11 \mathrm{~nm}$. The control signal collects in the entrance of a spectrometer that is equipped with infrared detectors. Fig. 5 shows a photo of the coater as well as the spectrometer of optical monitoring system.

The optical constants of materials such as $\mathrm{Si}, \mathrm{Ge} \mathrm{ZnS}, \mathrm{ZnSe}, \mathrm{SiO}, \mathrm{YbF}$, YF3 are determined by photometry at various process.

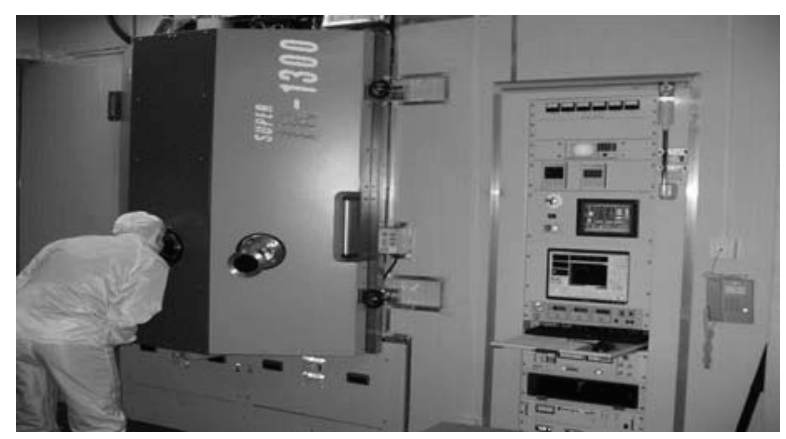

Fig. $5 \mathrm{~A}$ photo of the coater as well as the spectrometer

\section{Measured results}

The Fourier infrared spectrophotometer, JASCO FT/IR-460 is used for performance test, as shown in Fig.6. The specification of this spectrophotometer is as follows:

Wave-number accuracy: within $\pm 0.01 \mathrm{~cm}-1$

Resolution: $\quad 0.9,2,4,8,16 \mathrm{~cm}-1$

Signal-to-noise ratio: $\quad 15000: 1(4 \mathrm{~cm}-1$ at near $2200 \mathrm{~cm}-1)$

$100 \%$ T line flatness: $\quad$ within $100 \pm 1.0 \% \mathrm{~T}$. 


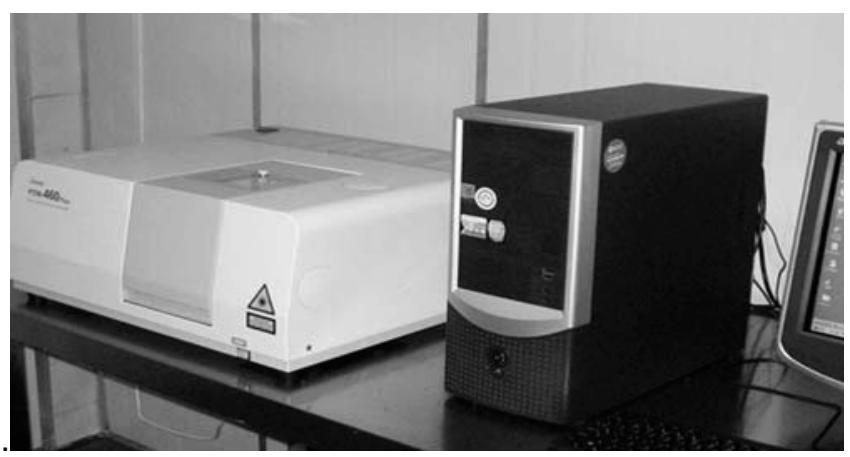

Fig.6 The photo of Fourier infrared spectrophotometer

Using this spectrophotometer, the properties of three kinds of infrared narrow band pass filters which are used in NIR, MIR \& FIR regions respectively are measured. The measured results are shown as in Fig.7.

\subsection{0umNBP Design \& Test Compare}
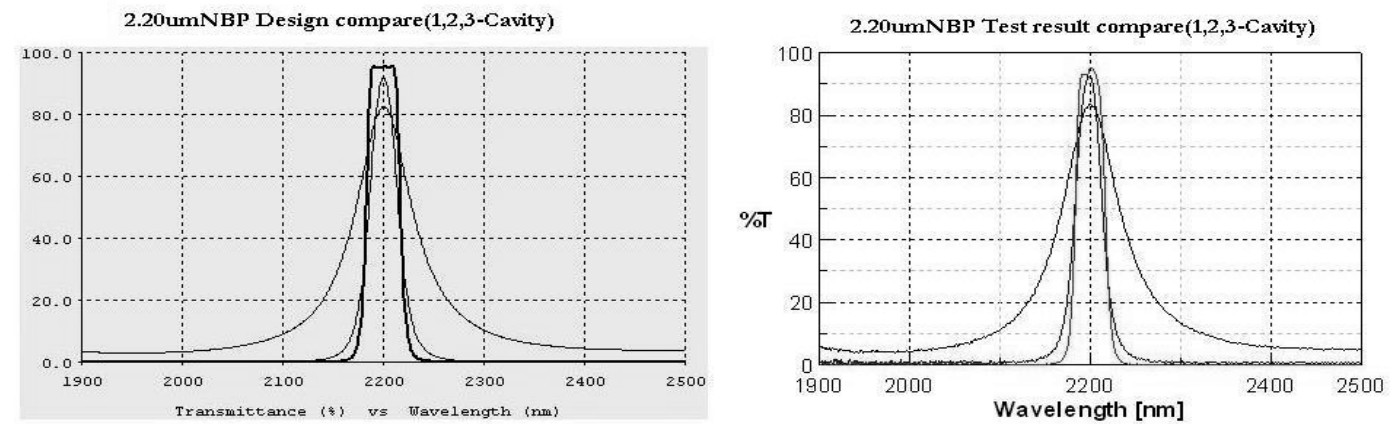

4.26umNBP Design \& Test compare
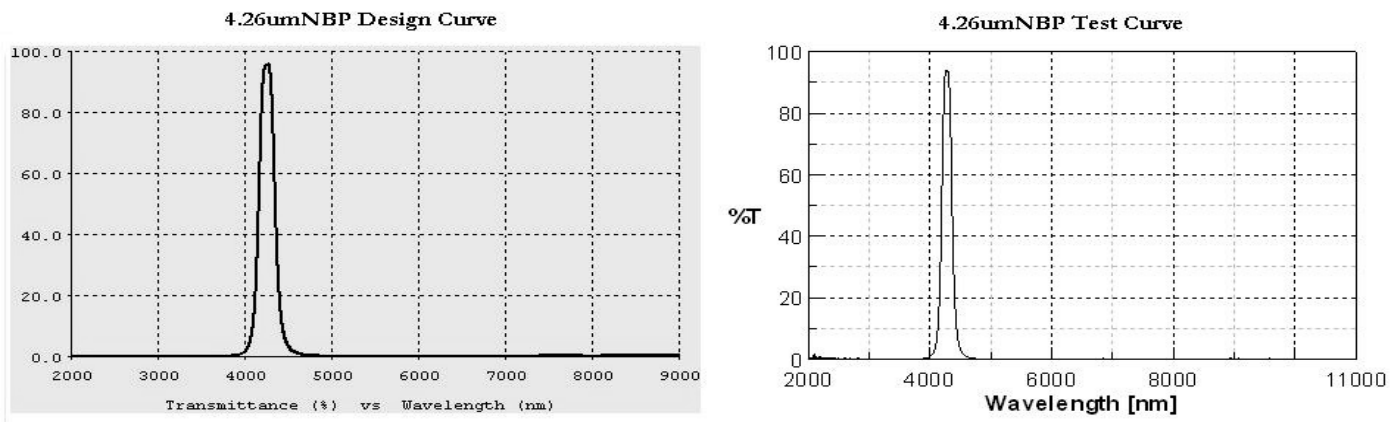

7.30umNBP Design \& Test compare
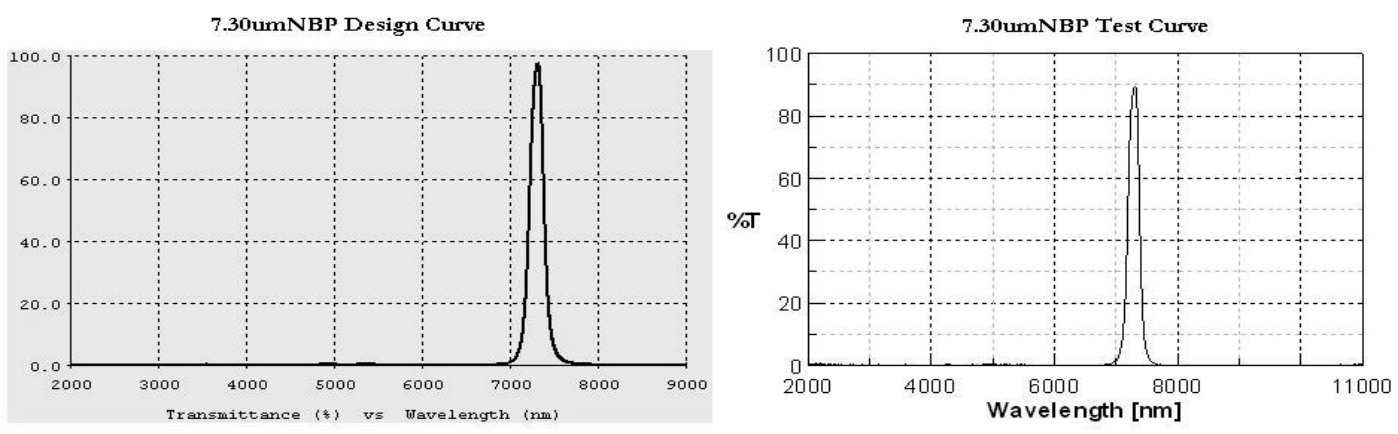

Fig.7 The measured results of three infrared filters with theoretical ones.

As shown in Fig.7, It can be seen that the measured results of the filters is good and in accordance 


\section{Conclusion}

Using method of matching the equivalent admittance, three kinds of infrared narrow band pass filters which are used in NIR, MIR \& FIR regions respectively are designed and manufactured. It can be proved that the measured performance of the filters has very good results in accordance with theoretical ones.

The important factors of manufacture of such high performance infrared narrow band pass filter are as followings:

(1) optimization design, including cavities design, coating material and substrate choose, as well as process design;

(2) accurate process control, such as process technology, parameter setup, and accurate control;

(3) suitably and stably equipment, especially, the coater was designed and modified to fit the requirement of stably technology process.

\section{References}

1. C.Grèzes-Besset1, N.Valette1, H.Krol1et.al., Multi-wavelength optical monitoring for infrared complex functions: Application to process improvement, Top meetings of Optical Interference Coatings ,2007,WC9

2. Frederic Lemarquis, Gerard Marchand, and Claude Amra, "Design and manufacture of low-absorption ZnS-YF3 antireflection coatings in the 3.5-16-mm spectral range," Appl. Opt. 37, 4239-4244(1998)

3. H.A.Macleod, Thin-Film Optical Filters, Third Edition, Institute of Physics Publishing Bristol and Philadelphia, p.293 